\title{
Antimicrobial stewardship: Improving antibiotic prescribing practice in a respiratory ward
}

\author{
Jing Ming Yeo
}

NHS Lothian, Edinburgh, Scotland, United Kingdom

\begin{abstract}
International efforts have mandated guidelines on antibiotic use and prescribing, therefore the focus is now on encouraging positive behavioral changes in antibiotic prescribing practice. Documentation of indication and intended duration of antibiotic use in drug charts is an evidencebased method of reducing inappropriate antibiotic prescribing. It is also a standard detailed in our local antimicrobial guidelines. We collected baseline data on compliance with documentation of indication and duration in drug charts in a respiratory ward which revealed compliance rates of $24 \%$ and $39 \%$ respectively. We introduced interventions to improve accessibility to the guideline and to increase awareness by distributing antibiotic guardian pocket cards with a three-point checklist and strategically-placed mini-posters. We also aim to increase team motivation by obtaining their feedback in multidisciplinary team meetings and by introducing certificates for their involvement in the quality improvement process. The results of the second cycle post-intervention showed an increase in compliance rates for documentation of indication and duration of $97 \%$ and $69 \%$ respectively. After a further awareness and discussion session at the multidisciplinary team meeting with the local antimicrobial management team audit nurses, a third cycle showed compliance rates of $94 \%$ and $71 \%$ for indication and duration respectively. This project has highlighted the importance of improving accessibility and of encouraging interventions that would bring about a change in personal value and subsequently in behavior and individual practice.
\end{abstract}

\section{Problem}

Antimicrobial stewardship is a concept comprising measures to improve the efficacy and reduce the adverse effects of antimicrobial use. This is achieved by reducing inappropriate antibiotic prescriptions which include unnecessary antibiotic use, unsuitable use of broad-spectrum antibiotics, and inappropriate dosage or duration of antibiotic use. This has been brought about by growing concerns over the issue of antimicrobial resistance which is currently a patient safety and public health concern.[1] Our local antimicrobial prescribing guideline (NHS Lothian Adult Antimicrobia Guidelines 2015) states that all antibiotic prescriptions should have the indication and intended duration documented in the drug chart. However, due to uncertainty, lack of awareness, and time constraints, there have been difficulties in adhering to the guidelines.

\section{Background}

An article in the British Medical Journal November 2014 edition entitled 'Antibiotic consumption must be cut' has highlighted the burden of inappropriate antibiotic prescribing in hospitals and in the community on the rising rate of antimicrobial resistance.[2] The concept of 'antimicrobial stewardship' was once again emphasised in the Royal Pharmaceutical Society's summit on antimicrobial resistance held on the 6th of November 2014 in London, urging care providers to take responsibility for their 'antimicrobial stewardship'. The article further emphasised the need to improve antibiotic prescribing practice across all health systems. Studies have shown that there is a higher likelihood of isolating antibioticresistance bacteria from patients who have received a higher number and duration of antibiotic courses.[3,4] Resistant organisms are associated with higher morbidity, mortality, and treatment costs, therefore antibiotic guidelines have been introduced across all health systems to reduce inappropriate antibiotic prescribing. Evidence-based methods to achieve this include the documentation of indication and intended duration of antibiotic use and the use of specimen culture results to narrow broad-spectrum therapies. Reducing over-prescribing of antibiotics would also reduce healthcare costs and antibiotic-associated healthcare-acquired infections. Furthermore, documentation of indication and duration would improve the quality of communication between doctors, nurses, and patients because it keeps the team informed of the plan for antibiotic use and of any intended reviews. This contributes to continuity of care.

\section{Baseline measurement}

We reviewed the drug charts of in-patients in the respiratory ward over a period of two weeks until we had sampled fifty in-patients who had been started on antibiotics during the current admission. Of the 83 antibiotic prescriptions in the 50 patients, only 20 prescriptions $(24 \%)$ had an indication documented in the drug chart, and only 32 prescriptions (39\%) had a duration or review date documented in the drug chart.

\section{Design}

We presented the results of the baseline study in the respiratory unit multidisciplinary team meeting and discussed measures that we can collectively take to improve the compliance rates. During the meeting, we encouraged the use of our local antimicrobial guideline 
(a resource available on our hospital intranet). We noted that it can be time-consuming to locate the antimicrobial guideline and a computer may not be readily available when the prescribing information is required. It is also safer to avoid relying on memory for the names of the first and second-line antibiotics and the duration recommended for the various infections. Therefore we designed pocket-sized antibiotic guardian cards (available to view under the supplementary file 'Antibiotic Guardian Pocket Card and Poster') which can be slotted behind name badges detailing these together with a reminder of the 3 points to consider when prescribing; (1) Antimicrobial Guidelines, (2) Indication and duration, (3) Send and check culture results. Other interventions suggested include strategically placed mini-posters (available to view under the supplementary file 'Antibiotic Guardian Pocket Card and Poster') containing relevant parts of the guideline placed in clinical areas where clinical notes are kept so that they are readily available during ward rounds. A further action agreed on was to document the indication and duration of antibiotic use on consultant ward round stickers in patients' clinical notes to keep team members aware of the plan for antibiotic use.

The sustainability of these interventions inherently hinges on instilling a personal value in prescribers to be antimicrobial guardians and stewards. The prescribers have to believe that the extra time it takes to look up the guideline and to write up the indication and duration are worthwhile efforts that have positive impacts on patients and on the healthcare system in which they work in. During the multidisciplinary team meetings, we highlighted issues of antimicrobial resistance on a global scale and the cost, mortality and trajectory of this healthcare issue to create a sense of urgency of the need for individual contributions. We also aim to increase external motivation and to express appreciation by providing certificates to prescribers as evidence of their involvement in this quality improvement process which can be mapped to their portfolio competencies.

\section{Strategy}

PDSA cycle 1:

We presented the results of the first cycle in the respiratory unit multidisciplinary team meeting and discussed issues surrounding inappropriate antibiotic use and antibiotic resistance to increase awareness. We distributed the antibiotic guardian pocket cards (available to view under the supplementary file 'Antibiotic Guardian Pocket Card and Poster') to members of the healthcare team and encouraged them to attach it to the back of their name badges, and placed posters (available to view under the supplementary file 'Antibiotic Guardian Pocket Card and Poster') in strategic areas to improve accessibility to the antimicrobial guideline. We encouraged junior team members to be a part of the quality improvement process by providing certificates that can mapped to their portfolio competencies. We also encouraged other healthcare team members to provide continuous instantaneous feedback to prescribers by alerting them to incompletely filled antibiotic prescriptions to allow prescribers to react to the information in a timely manner.
PDSA cycle 2:

In the second cycle carried out two months after the initial baseline result was presented, we were very fortunate to have the involvement of experienced audit nurses from our local antimicrobial management team who assisted with data collection. We presented the results of the second cycle at the respiratory unit multidisciplinary team meeting together with the antimicrobial management team audit nurses. As part of the awareness session, we additionally discussed several other issues surrounding antibiotic prescribing practice that could be improved upon including documentation of CURB-65 severity scoring, compliance of antibiotic choice with the local guideline, appropriate use of piperacillin-tazobactam, and criteria for intravenous to oral antibiotic switch.

PDSA cycle 3:

In the third cycle carried out two months after the second cycle, our data showed that the accessibility and awareness measures implemented have had a relatively sustained impact on antibiotic prescribing practices. We also noted that alongside our interventions, antimicrobial stewardship is a topic that is increasingly being stressed upon in academic articles and in the media including pledges taken by junior healthcare professionals on social media to be antibiotic guardians. We feel that there is a wave of awareness sweeping through healthcare which is having a powerful impact on improving antibiotic prescribing practices.

See supplementary file: ds6704.pdf - "Antibiotic Guardian Pocket Card and Poster"

\section{Post-measurement}

Compliance with documentation of indication in drug charts in the first, second, and third cycles were $24 \%$ (20 out of 83 antibiotic prescriptions), $97 \%$ (65 out of 67 antibiotic prescriptions), and $94 \%$ (68 out of 72 antibiotic prescriptions) respectively. Compliance with documentation of duration in drug charts in the first, second and third cycles were $39 \%$ (32 out of 83 antibiotic prescriptions), $69 \%$ (46 out of 67 antibiotic prescriptions), and $71 \%$ (51 out of 72 antibiotic prescriptions) respectively. The interventions and results are available to view as a flow chart in the supplementary file 'Antimicrobial Stewardship Quality Improvement Process'.

See supplementary file: ds6706.pdf - "Antimicrobial Stewardship Quality Improvement Process"

\section{Lessons and limitations}

We emphasized the use of one local guideline for clarity and to minimize confusion and errors. We simplified it into an antibiotic guardian-themed pocket card and made it specific for respiratory infections for use in a respiratory ward. A three-point checklist on the pocket card serves as a gentle reminder to document indication and duration, to send culture samples, and to check the results of sensitivities. We formally introduced the antibiotic guardian pocket 
card during the multidisciplinary team meeting to ensure that team members understand why they have been introduced and to obtain their feedback on it.

The meeting is an ideal forum for us to present our data and to discuss measures for improvement with various team members. We obtained their feedback for user-friendly interventions and engaged them in the process of creating an action plan. Senior team members act as natural role models and promoters of effective change and their support has greatly facilitated the team's acceptance of the need for an improvement in practice to take place. It would have been helpful to elect a senior team member as a respiratory unit antibiotic champion lead to act as a point of reference. We could also have used other discussion forums such as focus group meetings with junior team members to identify specific issues affecting junior members. The guidance, support and involvement of the local antimicrobial management team is also one of the most important aspects in carrying out an antibiotic prescribing quality improvement project. It would have been a greater advantage to involve them from the outset of the project.

Other limitations include a sense of a barrier in engaging healthcare professionals who are not prescribers. Nurses are an invaluable source of reducing antibiotic overuse as they are dispensing medications to patients daily and will therefore be alert to prescribing errors. A further effort that we could have done to engage non-prescribers would be to obtain their opinion on what they think prescribers could do to make prescriptions more transparent. We also noted that there are team members who are unable to attend the multidisciplinary team meetings when the discussions were carried out, therefore another means of distributing our data would have been useful, for example using email alerts and newsletters. There is also more work that could have been done to increase and sustain interest and awareness. For example, regular text alerts or notifications of interesting antimicrobial resistance-related statistics, antibiotic awareness activities such as the European Antibiotic Awareness Day, and recent exciting research such as point of care test kits to detect infections requiring antibiotic treatment.

\section{Conclusion}

This project aims to improve compliance with documentation of indication and duration of antibiotic use in line with our local antibiotic prescribing guidelines. Post-intervention results were encouraging with compliance rates increasing from $24 \%$ to $94 \%$ for indication, and from $39 \%$ to $71 \%$ for duration. With improvement in compliance with antibiotic prescribing guidelines, we hope to reduce antibiotic overuse and its side-effects. Overall, this project has highlighted the importance of improving accessibility, of encouraging collaboration between team members, and of encouraging interventions that would bring about a change in personal value and behaviour.

We hope that with increasing individual awareness of the weight of the antimicrobial resistance issue, antibiotic prescribing practice will continue to improve. We have also highlighted further interesting work to be done in the respiratory unit including improving compliance with antibiotic choice and with the criteria for intravenous to oral antibiotic switch. We hope to widen engagement with antibiotic guardian-related activities and to encourage the use of antibiotic guardian pockets cards in other medical and surgical wards via our local antimicrobial management team.

\section{References}

1 Ashiru-Oredope D, Sharland M, Charani E, McNulty C, Cooke J; ARHAI Antimicrobial Stewardship Group. Improving the quality of antibiotic prescribing in the NHS by developing a new Antimicrobial Stewardship Programme: Start Smart-Then Focus. J Antimicrob Chemother. 2012; 67 Suppl 1:i51-63.

2 Limb M. Antibiotic consumption must be cut. BMJ 2014; 349:96718

3 Goossens H, Ferech M, Vander Stichele R, Elseviers M; ESAC Project Group. Outpatient antibiotic use in Europe and association with resistance: a cross-national database study. Lancet. 2005; 365(9459):579-87.

4 Costelloe C, Metcalfe C, Lovering A, Mant D, Hay AD. Effect of antibiotic prescribing in primary care on antimicrobial resistance in individual patients: systematic review and meta-analysis. BMJ. 2010;340:c2096.

\section{Declaration of interests}

Nothing to declare.

\section{Acknowledgements}

Dr Tracey Bradshaw, Maeve Wheelhouse, Clare Gaston

\section{Ethical approval}

This work is deemed to be an improvement study and not a study on human subjects, therefore local policy excludes this from requiring ethical approval. 